\title{
SARS coronavirus outbreaks past and present-a comparative analysis of SARS-CoV-2 and its predecessors
}

\author{
Zheng Yao Low ${ }^{1} \cdot$ Ashley Jia Wen Yip ${ }^{1} \cdot$ Anshika Sharma $^{1} \cdot$ Sunil K. Lal ${ }^{1,2} \mathbb{D}$ \\ Received: 17 March 2021 / Accepted: 10 May 2021 / Published online: 1 June 2021 \\ (c) The Author(s), under exclusive licence to Springer Science+Business Media, LLC, part of Springer Nature 2021
}

\begin{abstract}
The Coronavirus Disease 2019 (COVID-19), a pneumonic disease caused by the SARS Coronavirus 2 (SARS-CoV-2), is the 7th Coronavirus to have successfully infected and caused an outbreak in humans. Genome comparisons have shown that previous isolates, the SARS-related coronavirus (SARSr-CoV), including the SARS-CoV are closely related, yet different in disease manifestation. Several explanations were suggested for the undetermined origin of SARS-CoV-2, in particular, bats, avian and Malayan pangolins as reservoir hosts, owing to the high genetic similarity. The general morphology and structure of all these viral isolates overlap with analogous disease symptoms such as fever, dry cough, fatigue, dyspnoea and headache, very similar to the current SARS-CoV-2. Chest CT scans for SARS-CoV-2, SARS-CoV and MERS-CoV reveal pulmonary lesions, bilateral ground-glass opacities, and segmental consolidation in the lungs, a common pathological trait. With greatly overlapping similarities among the previous coronavirus, the SARS-CoV, it becomes interesting to observe marked differences in disease severity of the SARS-CoV-2 thereby imparting it the ability to rapidly transmit, exhibit greater stability, bypass innate host defences, and increasingly adapt to their new host thereby resulting in the current pandemic. The most recent B.1.1.7, B.1.351 and P.1 variants of SARS-CoV-2, highlight the fact that changes in amino acids in the Spike protein can contribute to enhanced infection and transmission efficiency. This review covers a comparative analysis of previous coronavirus outbreaks and highlights the differences and similarities among different coronaviruses, including the most recent isolates that have evolved to become easily transmissible with higher replication efficiency in humans.
\end{abstract}

Keywords COVID-19 $\cdot$ SARS-CoV-2 $\cdot$ Coronavirus $\cdot$ Pandemic $\cdot$ Outbreak $\cdot$ Virus

\section{Introduction}

COVID-19 has affected the entire globe with more than $155,000,000$ infections worldwide [1] accompanied by a fatality rate of 3.4\% [1] and 3,250,000 associated deaths reported [2]. Contrary to the MERS-CoV and SARS-CoV outbreak believed to be associated with infected dromedary camel and bat reservoirs, the origin of SARS-CoV-2 to

Edited by Hartmut Hengel.

Zheng Yao Low and Ashley Jia Wen Yip have contributed equally.

Sunil K. Lal

sunil.1al@monash.edu

1 School of Science, Monash University, Sunway Campus, 47500 Bandar Sunway, Selangor DE, Malaysia

2 Tropical Medicine and Biology Platform, Monash University, Sunway Campus, 47500 Bandar Sunway, Selangor DE, Malaysia date is still unknown and debated, with sources suggesting that it may have originated from bats, akin to the previous SARS-CoV [3, 4]. The first human coronavirus outbreak was caused by the SARS-CoV in November 2002, originating from Guangdong Province, China, which rapidly progressed to Hong Kong, Beijing, Singapore, Vietnam, and Canada by March 2003 [5, 6]. The MERS-CoV outbreak on the other hand, was first reported from Jeddah, Saudi Arabia in September 2012 [7, 8]. Prior to the SARS-CoV outbreak (2002) there have been 4 other human infections of coronavirus by the HCoV-NL63, HCoV-HKU1, HCoV-229E, HCoV-OC43 isolates $[5,9]$. This makes the SARS-CoV-2 outbreak the 7th known infection of Coronaviruses in humans.

Coronaviruses are a well-known source of respiratory illness in humans [10-13]. It is the primary root for the common cold, manifesting as a mild illness, resulting in up to $20 \%$ of all common cold cases [14]. Coronaviridae (family) are zoonotic viruses, which are subdivided into four different genera: alpha, beta, gamma, and delta coronaviruses 
$[15,16]$. Among all the genera, the beta-coronavirus is documented to be responsible for severe illness and death in humans [17]. To date, there have been many identified hosts for the coronavirus, including rodents, feline, canine, turkey, swine, and humans, with bats serving as the primary reservoir with the exception of MERS-CoV reservoir being dromedary camels $[10,18]$. Being a beta-coronavirus, SARS-CoV-2 shares similar general morphology and structure as the other beta-coronavirus counterparts, albeit there are genetic and pathogenic differences which are described below. Another distinct feature of SARS-CoV-2 infections are the large numbers of asymptomatic cases in addition to patients with disease symptoms. Symptoms such as fever, dry cough, fatigue, dyspnoea, and headache are common to SARS-CoV-2, SARS-CoV and MERS-CoV infections [19]. Less common symptoms such as gastrointestinal discomfort, diarrhoea, conjunctivitis, skin discolouration or rashes, anosmia and dysgeusia have also been documented in SARS-CoV-2 infected patients with the latter two symptoms common in SARS and MERS-CoV patients [20, 21]. However, the multitude of asymptomatic infections for SARS-CoV-2 has been alarming and has contributed to the rapid spread of this pandemic [22]. An interesting case study by Chan et al. [22] reported a 10-year-old asymptomatic child having radiological bilateral ground-glass lung opacities similar to symptoms of SARS-CoV-2 infection however showed no symptoms of disease whereas, his family members were admitted to hospital upon clear symptoms like onset of fever, upper and lower respiratory tract symptoms and tested positive for SARS-CoV-2. SARS and MERS$\mathrm{CoV}$ infected patients also show close resemblance to this however the large number of asymptomatic patients showing similar lung opacities is much more common in SARSCoV-2 infected patients [23-25].

Like the SARS-CoV, the primary mode of SARS-CoV-2 transmission was reported to be via respiratory droplets $[3,4]$. However, apart from the usual direct droplet transmission, multiple studies have also indicated the possibility of fomite transmission, as the stability of SARS-CoV and MERS-CoV in faecal waste for a considerable time has been shown [26, 27]. A study suggests that the percentage of patients with positive faecal samples in SARS-CoV and MERS-CoV are $97 \%$ and $14.5 \%$ respectively [27]. Similar to its beta-coronavirus counterpart, the SARS-CoV-2 also showed viability in stool samples albeit at a much lower percentage at $55 \%$ as compared to the previous SARS-CoV [27]. Therefore, the possibility of faecal transmission for SARS-CoV-2 cannot be ruled out and more evidence needed to support the aforementioned for this rapidly evolving pandemic [26, 27].

Hence, it becomes important to understand and revisit the information on other Coronaviruses like the SARS-CoV and MERS-CoV and other related Coronaviruses that have successfully infected and caused disease to humans. With this approach in mind, many overlapping features and distinctions between the Coronaviruses listed above can provide insights into interim or long-term therapeutic solutions to effectively treat the current SARS-CoV-2 isolate.

\section{Comparative analysis of different coronavirus and SARS-CoV-2}

\section{Morphology and general structure}

In the past, the SARS-CoV and MERS-CoV have scored a significant $9.6 \%$ and $40 \%$ fatality rate, respectively $[4,6]$. Albeit having a lower estimated fatality rate at $3.4 \%$, the SARS-CoV-2 is progressing more quickly at a global scale and more infectious as compared to SARS-CoV and MERS$\mathrm{CoV}$. The rapid mutation in genomic context, especially the $\mathrm{S}$ protein in SARS-CoV-2 as compared to the previous SARS-CoV, renders the use of previous vaccines and antiviral therapeutic interventions ineffective. Hence, the study of viral morphology and pathogenesis play a pivotal role to identify the key features of SARS-CoV-2 with its similar counterpart, the SARS-CoV, to facilitate the drug repositioning progress, especially during these urgent times of need.

The general structure and genomic configuration of SARS-CoV-2 closely resemble the other coronaviruses, such as the SARS-CoV and MERS-CoV [10, 19]. The most prominent structure found in coronaviruses is the club-shaped spike projections on the virion surface. This structure gives the virus a crown-like appearance, which gives rise to the name, Coronavirus [15]. Coronavirus is the largest group of non-segmented positive-sense RNA viruses and is known to have an enveloped, spherical shape with a diameter of $65-125 \mathrm{~nm}$ in approximate $[18,28]$. Under the envelope, coronavirus possesses atypical helical and symmetrical nucleocapsids, rare amongst the positive-sense RNA virus [15]. The current SARS-CoV-2 consists of an RNA genome with a size of approximately 29.9 kilobases $(\mathrm{kb})$ [29]. The genome contains a typical 5' cap and a poly (A)-3'tail, which mimics an mRNA, granting it the ability to undergo translation [16]. Two-thirds of the viral RNA genome, starting from the 5' end, contains the open reading frame (ORF) $1 \mathrm{a}$ and $1 \mathrm{~b}$ genes which primarily encode a large array of non-structural proteins (NSPs). The NSPs (nsp 1-16) are known to aid in viral replication $[11,18]$. The remaining one-third of the genome encodes for structural proteins, a common feature for all coronaviruses. The structural proteins encoded by the ORF at the 3' end include the spike protein (S), envelope protein $(\mathrm{E})$, membrane protein $(\mathrm{M})$ and nucleocapsid protein (N) $[11,16]$.

The SARS-CoV-2 consists of 13-15 open reading frames (ORFs) containing 30,000 nucleotides [30]. The typical 
general layout of the SARS-CoV-2 genome is as follows [5'-leader-UTR-replicase-S-E-M-N-3'-UTR-poly (A) tail], with the accessory genes scattered between the structural genes at the $3^{\prime}$ end of the genome [16, 30].

\section{The genetic constitution and possible origin of the SARS-CoV2}

The beta-coronaviruses can be subdivided into four lineages: A, B, C and D [31]. Several notable examples of beta coronaviruses include $\mathrm{OC} 43$ and HKU1 from lineage A that were discovered in 1967 and 2005, respectively [5]. SARS-CoV and the present SARS-CoV-2 are both categorized under lineage $B[6,9,32]$, whereas MERS-CoV belongs to lineage $\mathrm{C}[4,8,33]$. To date, lineage $\mathrm{D}$ beta coronaviruses have only been reported in bats, such as the Rousettus bat coronavirus (Ro-BatCoV HKU9) [34]. Several theories revolve around the origin of SARS-CoV-2. Possible explanations include natural selection in humans after zoonotic transfer and natural selection in animal reservoirs prior to zoonotic transfer [3]. Yet, for a significant natural selection to occur, a larger animal population density is required and angiotensinconverting enzyme (ACE-2) encoding gene homologous to human ortholog must be present in the animal host [35]. Wu et al. and Guo et al. proposed another viewpoint, which states that the virus evolved to its pathogenic state prior to zoonotic transfer $[23,36]$.

SARS-CoV-2 was suggested to be genetically more closely related to SARs-related coronavirus (SARSr-CoV) than the MERS-CoV. The (SARSr-CoV) is a species of enveloped positive-sense single-stranded RNA virus that enters its host cell by binding to the angiotensin-converting enzyme 2 (ACE2) receptor. The SARSr-CoV has multiple strains which are of bat-related coronavirus, in which includes the Bat SARS-like coronavirus WIV1 (Bat SLCoV-WIV1), Bat coronavirus RaTG13, SARS-CoV and many more [37]. A genome sequence similarity of $96.2 \%$ between SARS-CoV-2 and Bat CoVER aTG13 was observed through full-length sequencing of the short region of RNAdependent RNA polymerase (RdRp) [19]. When compared to bat derived SARS-like CoV (bat-SL-CoVZC45, MG772933.1), a nucleotide similarity of $86.9 \%$ was reported [10]. On the other hand, SARS-CoV-2 has been reported to share $79 \%$ and $50 \%$ genetic similarity to SARS-CoV and MERS-CoV, respectively [38]. The SARS-CoV-2 possesses a $94.4 \%$ similarity in the amino acid sequences of the seven conserved replicase domains in the ORF1ab when compared to SARS-CoV. Under the same study, an $87.1 \%$ sequence matched between SARS-CoV-2 with several SARSr-CoV sequences were revealed via next-generation sequencing [19]. Interestingly, there was a $93.1 \%$ nucleotide similarity of receptor binding spike protein encoded by $S$ gene between SARS-CoV-2 and bat coronavirus (BatCoV RaTG13). This result is in line with similar studies where matching of $87.2 \%$ to $83.9 \%$ in $\mathrm{S}$ protein to minimal receptor-binding domain were demonstrated between the SARS-CoV-2 and the previous SARS-CoV [39]. As SARS-CoV-2 is highly similar to the bat coronavirus, bats were suggested to be the reservoir host for the ancestor of SARS-CoV-2 and subsequently transmitted to humans through an unrevealed intermediate host.

Albeit the aforementioned RaTG13 from Rhinolophus affinis bats having high genetic similarity index of $96 \%$ with the SARS-CoV-2 [3], the Malayan pangolins (Manis javanica) was reported to have a higher genetic similarity index in all six major receptor-binding domain residues [40, 41]. In the past, cleavage sites were only described in low pathogenicity avian influenza virus but absent in bat and pangolin beta-coronaviruses [35, 42]. Interestingly, the distinct cleavage site in SARS-CoV-2 was reported to be similar to several bird flu strains which transmit easily among humans [19]. Therefore, by conducting retrospective serological studies and studying banked human samples could potentially aid in understanding whether such spread had occurred for this current pandemic [43]. Based on the aforementioned above, such as the $79 \%$ genetic similarity between SARS-CoV-2 and SARS-CoV, 94.4\% amino acid sequence similarity from the seven conserved replicase domains in the ORF1ab between SARS-CoV-2 and SARS-CoV, 87.1\% matched sequence between SARS-CoV-2 and SARS-CoV and many more, strongly suggests that the SARS-CoV-2 are much more akin to the previous SARS-CoV than the MERS-CoV [19, 38] (Fig. 1, Table 1).

\section{The key distinctions and features of SARS-CoV-2, SARS-CoV and MERS-CoV}

\section{Receptor recognition}

The SARS-CoV and SARS-CoV-2 possess the same host cell surface receptor-the ACE2 protein. However, the SARS-CoV-2 possesses a higher binding affinity compared to SARS-CoV attributed to a single mutation at N501T in the Spike (S) protein [40, 46, 47]. Besides, a study has also revealed a large protein interaction surface with high binding-affinity between SARS-CoV-2 and ACE-2 receptors at 18 interactions, compared to only 8 interactions between SARS-CoV and ACE2, amounting up to 15 -fold stronger interaction in SARS-CoV-2 [48]. The same study has also illustrated strong multi-epitope synapse adhesion between spike protein (amino acid position 471-486, 496-505, 404-416 \& 446-456) in SARS-CoV-2 and human ACE-2 receptor, contributing to a strong viral surface-host's epithelial adhesion, suggesting the need for multi-epitope high affinity antibodies for antiviral therapeutics as compared to SARS-CoV [48]. This is also evident in the past when 


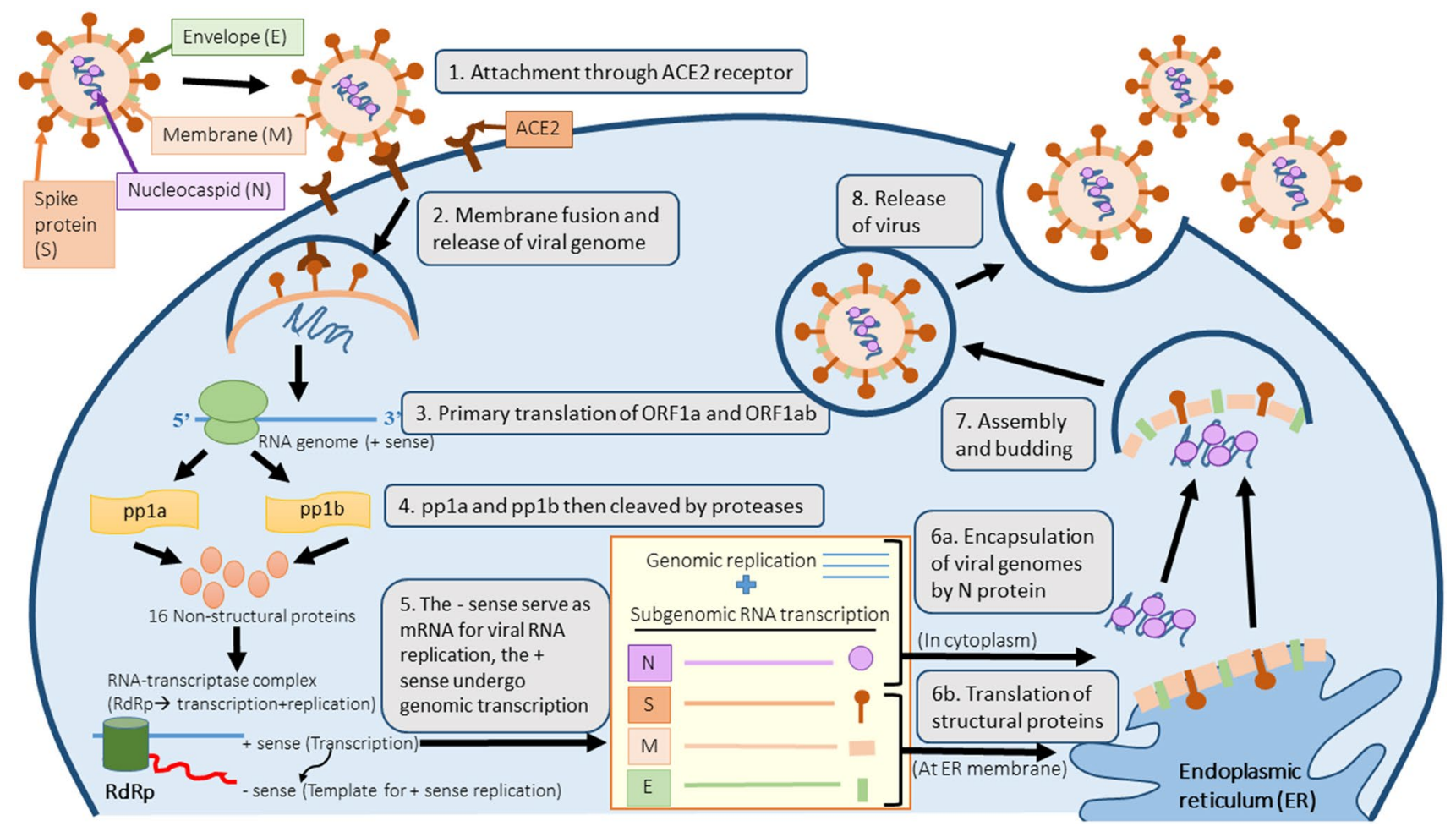

Fig. 1 The replication cycle of SARS-CoV-2. The attachment and entry of the SARS-CoV-2 are mediated by the S, or "spike" surface glycoprotein [44]. The S protein can be subdivided into S1 receptor binding domain and S2 fusion domain [44]. Upon proteolytic activation by the host cells, the $\mathrm{S}$ proteins bind to the cellular receptor, angiotensin-converting enzyme-2 (ACE-2), which facilitates membrane fusion with host cell membrane through the endocytosis, leading to the subsequent release of viral RNA genomes in the host cytoplasm. Both co-terminal polyproteins, pp1a and $\mathrm{pp} 1 \mathrm{~b}$, produced from the translation of ORF1a and ORF1b are cleaved by proteases

studies were done on combination of two monoclonal antibodies, CR3022 + CR3014, is much more effective in neutralizing SARS-CoV via the interaction with S2 domain of SARS-CoV S protein than CR3022 or CR3014 alone [49]. This gives a strong indication where one antibody may not be sufficient in hindering the already stronger affinity from SARS-CoV-2. Hence, this highlights the need for multiple multi-epitope high affinity antibodies that targets different sites of adhesion synapse in passive immunisation for COVID-19, to provide strong antiviral response [48].

Apart from the aforementioned above, other SARSCoV-2 variants (B.1.1.7, B.1.351 \& P.1) have also shown various spike protein mutations (N501Y, P681H, K417N, E484K and K417T), concomitantly enhancing binding affinity of S protein for human ACE-2 receptor to a greater extent.

The B.1.1.7 (20I/501Y.V1, VOC 202012/01) variant, B.1.351(20H/501Y.V2) variant, and the P.1 variant (B.1.1.28 subclade) have been described following viral genome sequencing. The most prominently identified mutations to form non-structural proteins (nsps). These nsps will assemble into a replicase-transcriptase complex (RTC), which encodes for many enzymes such as RNA-dependent RNA polymerase (RdRp). During replication, RdRp will produce negative-sense RNA copies to serve as templates for replications of positive-sense RNA genomes. Then, a series of subgenomic mRNAs is produced during transcription and translated into respective viral proteins, which will be further assembled and packaged at respective sites. Following that, the resulting virion will undergo budding and release of virions through exocytosis

between all variants lie within the spike (S) proteins [50]. The B.1.1.7 mutation was first identified in Denmark, United Kingdom and Northern Ireland in December 2020. Differ from the original variant, the B.1.1.7 variant carries a mutation in the $\mathrm{S}$ protein, an amino acid change from asparagine to tyrosine at position $501(\mathrm{~N} 501 \mathrm{Y})$ in the receptor-binding domain of the spike protein, which increases the binding affinity to human ACE-2 receptor [50,51]. Also, another mutation in the $\mathrm{S}$ protein, an amino acid change from proline to histidine at position $681(\mathrm{P} 681 \mathrm{H})$ in the spike protein, one of the four residues that creates a furin cleavage site between $\mathrm{S} 1$ and $\mathrm{S} 2$ in spike has been shown to promote entry into respiratory epithelial cells and transmission in animal models. Apart from that, a deletion of 2 amino acids at positions 69 and 70 (del 69-70) in the S protein provides an evasion point, S-gene target failure (SGTF) in RT-PCR assay, inadvertently increasing false-negative results and hidden transmissibility [50].

Moving on to the next variant, the B.1.351 was first detected in Nelson Mandela Bay, South Africa in December 
Table 1 Characteristic features of SARS-CoV-2, SARS-CoV and MERS-CoV

\begin{tabular}{|c|c|c|c|}
\hline & SARS-CoV-2 (COVID-19) & SARS-CoV & MERS-CoV \\
\hline Origin & $\begin{array}{l}\text { China } \\
\text { Source: Unknown, possible bats }\end{array}$ & $\begin{array}{l}\text { China } \\
\text { Source: horseshoe bats }\end{array}$ & $\begin{array}{l}\text { Saudi Arabia } \\
\text { Source: dromedary camels }\end{array}$ \\
\hline Year of Outbreak & Dec 2019-ongoing & 2002-2004 & 2012-ongoing \\
\hline $\begin{array}{l}\text { Number of reported countries } \\
\text { affected }\end{array}$ & > 190 (Ongoing) & 26 & 27 \\
\hline $\begin{array}{l}\text { Reproductive number/Transmis- } \\
\text { sibility }\left(\mathrm{R}_{0}\right)\end{array}$ & $\sim>3^{*}$ & $\sim 2^{*}$ & $\sim 0.7^{*}$ \\
\hline Genera & Beta-coronavirus & Beta-coronavirus & Beta-coronavirus \\
\hline Lineage & B & B & $\mathrm{C}$ \\
\hline Common symptoms & $\begin{array}{l}\text { Fever, cough, lower respiratory } \\
\text { tract infection }\end{array}$ & $\begin{array}{l}\text { Fever, cough, lower respiratory } \\
\text { tract infection, diarrhoea, runny } \\
\text { nose }\end{array}$ & $\begin{array}{l}\text { Fever, cough, lower respiratory } \\
\text { tract infection, diarrhoea, runny } \\
\text { nose }\end{array}$ \\
\hline Mode of transmission & $\begin{array}{l}\text { Close contact with an infected } \\
\text { individual or via respiratory } \\
\text { droplets }\end{array}$ & $\begin{array}{l}\text { Close contact with an infected } \\
\text { individual or via respiratory } \\
\text { droplets }\end{array}$ & $\begin{array}{l}\text { Close contact with an infected } \\
\text { animal and individual or via } \\
\text { respiratory droplets }\end{array}$ \\
\hline Receptor Recognition & ACE-2 & ACE-2 & DPP4/CD26 \\
\hline Incubation period & $\sim 2-14$ days* [45] & 2-7 days [45] & 2-14 days [45] \\
\hline Preferred detection method & $\begin{array}{l}\text { Real-time quantitative reverse- } \\
\text { transcription PCR (RT-PCR) }\end{array}$ & $\begin{array}{l}\text { RT-PCR, Enzyme-linked immu- } \\
\text { nosorbent assay (ELISA) }\end{array}$ & $\begin{array}{l}\text { RT-PCR, serum test for antibodies } \\
\text { targeting MERS virus }\end{array}$ \\
\hline Clinical examination & $\begin{array}{l}\text { Chest-CT } \\
\text { (Bilateral ground-glass opacities) }\end{array}$ & $\begin{array}{l}\text { Chest-CT } \\
\text { (Numerous infiltrative patchy } \\
\text { shadows) }\end{array}$ & $\begin{array}{l}\text { Chest-CT } \\
\text { (Extensive ground-glass opacities) }\end{array}$ \\
\hline Reported cases & $>155,000,000^{* *}$ & $\sim 8000[6]$ & $>2564[30]$ \\
\hline Reported death & $>3,250,000 * *$ & $\sim 774[6]$ & $>881[30]$ \\
\hline Fatality rate & $\sim 3.4 \%$ (initial estimate) & $\sim 9.6 \%$ & $\sim 40 \%$ \\
\hline
\end{tabular}

*The reproductive number $\left(\mathrm{R}_{0}\right)$ varies across different countries and regions, the given values in the table are based on the few cited sources in this manuscript. The incubation period for COVID-19 in Table 1 was obtained from the CDC on 20 February 2021, the value is uncertain as the pandemic is still ongoing [45]

**The associated values for COVID-19 vary across time as the pandemic is still on-going at a rapid pace at the time of this writing. (May 6, 2021)

2020. Apart from the similar N501Y mutation found in the B.1.1.7 variant, this variant has two distinct mutations in the S protein (K417N, E484K, and N501Y) [52]. The K417N mutation on the RBD of S protein interacts with the D30 ACE-2 protein residue, contributing to significant enhancement of binding affinity to human ACE- 2 receptors. Besides, there have been reports on the likelihood of K417N mutation towards the abolishment of key interactions with class 1 neutralising antibodies, contributing towards immune evasion [52]. Apart from that, the E484K mutation in S protein interacts with the K31 human ACE-2 protein residues, enhancing the binding affinity with the receptor [52, 53]. Furthermore, the E484K mutation has been shown to reduce neutralization by convalescent sera and monoclonal antibodies, leading to reinfection cases in Brazil [50].

The variant P.1, a subclade of B.1.1.28 variant was first identified in Tokyo, Japan in January 2021 by the National Institute of Infectious Disease (NIID). This lineage contains the usual N501Y spike protein mutation found in other variants in addition to two other S protein mutations (K417T, E484K, and N501Y) [54]. Apart from the aforementioned roles for E484K and N501Y S mutations, the K417T spike protein mutation might be playing a significant role in this variant. Given that it is a very recent discovery, not much is known about this variant, however, there is evidence suggesting that the P.1 variant may affect the ability of antibodies to recognize and neutralize the virus [54].

An interesting similarity was also found across these three emerging variants where D614G mutation in S protein is consistent across the variants, increasing the binding affinity for ACE-2 receptor [50]. The D614G mutation denotes an amino acid change from aspartic acid to glycine at position 614 in the S protein, leading to reduced S1 shedding and increased total S protein incorporated into the virion [50]. However, given that there are different mutations across the different emerging SARS-CoV-2 variants, vaccines were found to be effective against all these mutations. As such, the Pfizer-BioNtech BNT162b2 vaccine has successfully induced neutralisation against the panel of the aforementioned spike protein mutations across different SARS-CoV-2 variants [55]. 
Besides, differing from SARS-CoV, SARS-CoV-2 has three short insertions in the $\mathrm{N}$-terminal domain along with distinct 4/5 key residues in the ACE-2 receptor-binding motif [19]. MERS-CoV, on the other hand, recognises host dipeptidyl-peptidase 4 (DPP4)/CD26 receptor. Apart from that, SARS-CoV-2 has also been reported to have gained a polybasic cleavage site at the $\mathrm{S} 1-\mathrm{S} 2$ boundary in the spike protein with an insertion of 12 nucleotides, which was not observed in SARS-CoV. The insertion of these nucleotides is predicted to provide three O-linked glycans which are believed to involve in the immune-evasion [46, 47]. Numerous cases of aggressive COVID-19 progression in hypertensive and diabetic patients have been reported post renin-angiotensin-aldosterone system (RAAS) inhibitor administration. Such inhibitors disrupt RAAS and subsequently increase ACE2 levels thus, resulting in the rapid progression of COVID-19, further indicating the correlation between levels of host ACE2 and COVID-19 disease progression $[47,56]$ (Table 2).

\section{Attachment and entry}

The $\mathrm{S} 1$ receptor binding domain of the spike protein is the same across the different coronaviruses (SARS-CoV-2, MERS-CoV and SARS-CoV), utilizing the S1-C terminal (S1-CTD) [57]. However, only 64\% similarity has been reported in the S1-CTD when compared to SARS-CoV [63]. In addition, the $\mathrm{S} 2$ spike protein fusion domain in SARSCoV-2 showed a $90 \%$ identity with respect to SARS-CoV [63]. There are generally four different modes of proteolytic activation of coronavirus spike proteins, including the proprotein convertases, extracellular proteases, cell surface proteases and lysosomal proteases. The three latter modes are involved in the activation of SARS-CoV spike proteins, including extracellular protease (elastase), cell surface proteases (TMPRSS2) and lysosomal proteases (cathepsin
L/B) [44, 57, 61]. However, it has been reported that SARSCoV-2 uses all four modes of proteolytic activation, which includes Proprotein convertases (Furin) [46, 47, 64]. On the other hand, the MERS-CoV utilises Proprotein convertases (Furin), Cell surface proteases (TMPRSS2) and Lysosomal proteases (Cathepsin L) [46, 57]. Furin is highly expressed in the human lungs, hence it may be playing a key role in enhancing the infectivity of SARS-CoV-2 in the human lungs thus, allowing increased exploitation of the host as compared to the SARS-CoV [46, 47, 64]. Apart from that, the ability to exploit four different modes of proteolytic activation might confer an infectivity advantage for SARSCoV-2 compared to the SARS-CoV and MERS-CoV, in which both uses only three modes. It was also found that SARS-CoV-2 is more susceptible to furin cleavage, as compared to the SARS-CoV [64].

\section{Viral replication and genetic expression}

The pp1a and pp1b, produced from the translation of ORF1a and ORF $1 \mathrm{~b}$ are cleaved by proteases to form non-structural proteins (NSPs). The NSPs assemble into a replicase-transcriptase complex (RTC), which encodes for many enzymes such as the RNA-dependent RNA polymerase (RdRp). The significant difference between the SARS-CoV-2 and SARS$\mathrm{CoV}$ genetic expression is the absence of $8 \mathrm{a}$ protein and changes in the number of amino acids in $8 \mathrm{~b}$ and $3 \mathrm{c}$ protein [28]. Another key difference between the SARS-CoV-2 compared to SARS-CoV, is the transmembrane helical segments in the ORF1ab encoding for NSP2 and NSP3 [65]. The study has reported that at amino acid position 501 (position 321 in NSP2), the SARS-CoV-2 encodes for glutamine residue instead of threonine residue found in the SARS-CoV [65]. With this substitution, the side chain now has a higher polarity and stronger ability to form hydrogen bonds (H-bonds), providing stability to the protein. Also, the same study

Table 2 A summary of receptor recognition from different coronavirus

\begin{tabular}{|c|c|c|c|}
\hline Coronavirus genera & Identified coronavirus & Receptor recognition & Source \\
\hline \multirow[t]{4}{*}{ Alpha $(\alpha)$} & Human Coronavirus NL63 (HCoV-NL63) & $\begin{array}{l}\text { Angiotensin-converting enzyme-2 (ACE2) } \\
\text { Aminopeptidase N (APN) }\end{array}$ & {$[15,57]$} \\
\hline & Human Coronavirus 229E (HCoV-229E) & Aminopeptidase N (APN) & {$[15,57]$} \\
\hline & Porcine Transmissible Gastroenteritis Coronavirus (TGEV) & Aminopeptidase N (APN) & {$[15,57]$} \\
\hline & Canine coronavirus $(\mathrm{CCoV})$ & Aminopeptidase N (APN) & {$[15]$} \\
\hline \multirow[t]{4}{*}{$\operatorname{Beta}(\beta)$} & Severe Acute Respiratory Syndrome Coronavirus (SARS-CoV) & Angiotensin-converting enzyme-2 (ACE2) & {$[57-60]$} \\
\hline & $\begin{array}{l}\text { Severe Acute Respiratory Syndrome Coronavirus-2 (SARS- } \\
\text { CoV-2) }\end{array}$ & Angiotensin-converting enzyme-2 (ACE2) & {$[40,60,61]$} \\
\hline & Middle East Respiratory Syndrome Coronavirus (MERS-CoV) & Dipeptidyl-peptidase 4 (DPP4)/CD26 & {$[57,58,60]$} \\
\hline & Mouse Hepatitis Coronavirus (MHC) & $\begin{array}{l}\text { Carcinoembryonic antigen-related cell } \\
\text { adhesion molecule } 1 \text { (CAECAM1) }\end{array}$ & {$[57,58]$} \\
\hline $\operatorname{Gamma}(\gamma)$ & Avian Infectious Bronchitis Coronavirus (IBV) & $\alpha-2,3$ linked sialic acids & {$[57]$} \\
\hline Delta $(\delta)$ & Porcine deltacoronavirus (PdCV) & porcine-APN (pAPN) & [62] \\
\hline
\end{tabular}


reported that at amino acid position 723 (position 543 in NSP3), SARS-CoV-2 encodes a serine residue instead of a glycine as per in SARS-CoV. This substitution may lead to an increase in local stiffness of the polypeptide chain and increased ability to form $\mathrm{H}$-bonds, affecting enzyme active sites [65]. In addition, at the amino acid position 1010 (position 192 for NSP3), SARS-CoV-2 encodes for proline instead of isoleucine, as found in SARS-CoV [65]. With proline in place for isoleucine, a steric bulge will be expected, providing stiffness to the molecular structure of the SARSCoV-2 [65] (Table 3).

\section{The immune-response towards SARS-CoV-2 infection}

In general, the human body has a few lines of defences to fend off virus, bacteria, and other microbes. The first line of defence (also known as the innate immunity) provides an initial defence against the infection. There are a range of first line barriers which includes the phagocytes, dendritic cells, natural killer cells, complement cells to the simplest epithelial barrier. The immune response for any viral infection is generally the adaptive immunity, in which generated by the white blood cells known as the "lymphocytes", that entails the $\mathrm{B}$ cells and $\mathrm{T}$ cells. The humoral response or also known as the antibody-mediated response plays an important role for antibody production to neutralize the viral antigen. Lymphocytes primarily reside in the lymph nodes and spleens [66]. Upon SARS-CoV-2 invasion, the foreign antigen presented by the SARS-CoV-2 drives the primary antibody (IgM antibody production) and secondary antibody response (IgG antibody production) from B cells, which ultimately increases the levels of several inflammatory cytokines for subsequent defence against viral invasion [67]. The released cytokines include the TNF- $\alpha$, IL- 1 and IL- 6 which stimulate the CD4+ and CD8+ T-cells. The CD4+ T cells help to coordinate the immune response via stimulation of other immune cells, such as macrophages, B cells, and CD8+ T killer cells to clear the pathogen and destroy the infected cells [68]. However, in certain scenarios, a notable reduction of CD4+ and CD8+ T cells can be seen in severe cases at less than 800 , and 400 cells $/ \mu \mathrm{L}$ respectively due to $\mathrm{T}$ cells exhaustion, concomitantly leads to collapse in host immunity defence. This was contributed by the persistent stimulation of the virus, which enhances the production of IL-10 inhibitory cytokine in the body [69].

Immunoglobulin $\mathrm{M}(\mathrm{IgM})$ is commonly known to provide most of the primary antibody defence upon viral infections. On the other hand, the high-affinity IgG is tagged as a secondary antibody immune response that is vital for longterm immunity and immunological memory [70]. Hence, in the event of SARS-CoV-2 infection, IgM is often released in large amounts 3-6 days post-infection followed by $\mathrm{IgG}$

Table 3 The key distinctions and features of SARS-CoV-2, SARS-CoV and MERS-CoV

\begin{tabular}{|c|c|}
\hline eceptor recognition & $\begin{array}{l}\text { SARS-CoV and SARS-CoV-2 utilises host angiotensin-converting enzyme (ACE2) } \\
\text { SARS-CoV-2 has much higher binding affinity compared to SARS-CoV due to single mutation at N501T in the Spike (S) } \\
\text { protein [40, 46, 47]. A study has also revealed a large protein interaction surface (18 interactions) with high binding- } \\
\text { affinity between SARS-CoV-2 and ACE-2 receptors compared to SARS-CoV ( } 8 \text { interactions), amounting up to 15-fold } \\
\text { stronger interaction in SARS-CoV-2 [48] } \\
\text { Other SARS-CoV-2 variances (B.1.1.7, B.1.351 \& P.1) have also shown various spike protein mutations (N501Y, P681H, } \\
\text { K417N, E484K and K417T), in which enhances the binding affinity of S protein for ACE-2 receptor at a greater extent } \\
\text { [51-55] } \\
\text { SARS-CoV-2 has three short insertions in the N-terminal domain along with distinct } 4 / 5 \text { key residues in the ACE-2 } \\
\text { receptor-binding motif which is not present in SARS-CoV [19] } \\
\text { Unlike SARS-CoV, the SARS-CoV-2 gained a polybasic cleavage site at the S1-S2 boundary in the spike protein with an } \\
\text { insertion of } 12 \text { nucleotides to provide three O-linked glycans involved in immunoevasion [46, 47] } \\
\text { MERS-CoV, on the other hand, recognises host dipeptidyl-peptidase } 4 \text { (DPP4)/CD26 receptor }\end{array}$ \\
\hline Attachment \& entry & $\begin{array}{l}\text { The SARS-CoV-2 shows only } 64 \% \text { similarity in S1-CTD when compared to SARS-CoV [63] } \\
\text { The S2 spike protein fusion domain in SARS-CoV-2 showed a 90\% identity with respect to SARS-CoV [63] } \\
\text { The SARS-CoV-2 uses } 4 \text { modes of proteolytic activation, including the Proprotein convertases (Furin) [46, 47, 64]. Furin } \\
\text { is highly expressed in the human lungs, hence allowing enhanced infectivity of SARS-CoV-2 in the human lungs for } \\
\text { increased exploitation compared to the SARS-CoV [46, 47, 64] } \\
\text { The SARS-CoV-2 is more susceptible to furin cleavage, as compared to the SARS-CoV [64] } \\
\text { On the other hand, the MERS-CoV also utilises Proprotein convertases (Furin), in addition with Cell surface proteases } \\
\text { (TMPRSS2) and Lysosomal proteases (Cathepsin L) [46, 57] }\end{array}$ \\
\hline $\begin{array}{l}\text { Viral replication and } \\
\text { genetic expression }\end{array}$ & $\begin{array}{l}\text { The SARS-CoV-2 genetic expression showed changes in the number of amino acids in } 8 \mathrm{~b} \text { and } 3 \mathrm{c} \text { protein and an absence } \\
\text { of } 8 \text { a protein as compared to the SARS-CoV [28] } \\
\text { The SARS-CoV-2 encodes for glutamine residue instead of threonine residue found in the SARS-CoV at amino acid } \\
\text { position } 510 \text { [65]. At amino acid position 723, the SARS-CoV-2 encodes for serine residue instead of glycine found in } \\
\text { SARS-CoV [65]. In addition, at amino acid position 1010, SARS-CoV-2 encodes for proline instead of isoleucine, as } \\
\text { found in SARS-CoV [65] }\end{array}$ \\
\hline
\end{tabular}


antibody production, which may be detected 8 days after the onset of symptoms [70, 71]. A study has shown that SARS$\mathrm{CoV}-2$ leads to significant reduction of $\mathrm{Bcl}-6^{+} \mathrm{T}$ follicular helper cells $\left(\mathrm{Bcl}-6^{+} \mathrm{T}_{\mathrm{FH}}\right)$ and absence of germinal center $\mathrm{B}$ cells in lymph nodes, contributing to dysregulated humoral response. This was due to the interference on the production of high affinity pathogen specific antibodies, leading to impaired $\mathrm{IgG}$ response. Apart from that, the study has also observed B lymphopenia in COVID-19 patients, in which might be a contribution of excess TNF- $\alpha$ production which suppresses differentiation in Bcl- $6^{+} \mathrm{T}_{\mathrm{FH}}$ and germinal center loss, concomitantly compromises the production of quality $\mathrm{IgG}$ antibodies [72].

Another study conducted on the avidity maturation of IgG antibody upon SARS-CoV-2 infection also highlights an incomplete maturation, low or intermediate avidity in $\mathrm{IgG}$ to receptor binding domain (RBD) [66]. In which, the binding strength between $\operatorname{IgG}$ and its epitope, and strength of the multivalent interactions where both antigen interacting with multiple IgG antigen-binding sites greatly reduced [66]. Besides that, the avidity maturation of the IgG antibody allows the differentiation of acute and past SARS-CoV-2 infection, albeit not completely certain [73]. Bauer and colleagues stated that low avidity of $\mathrm{IgG}$ antibody illustrate an acute sars-cov-2 infection, intermediate avidity $\operatorname{IgG}$ might indicate recent infection and high avidity IgG might indicate a past infection [74]. Therefore, the importance of antibody immune response, especially the levels, affinity, and avidity of IgG (Immunoglobulin G) towards SARS-CoV-2 infection cannot be overlooked.

\section{The transmission and stability of SARS-CoV-2 vs SARS-CoV}

To contain the SARS-CoV-2, the reproduction number becomes crucial. The basic reproduction number $\left(\mathrm{R}_{0}\right)$ is an important metric to measure the potential of a disease spread within a completely susceptible population [75]. For instance, if a disease has an $R_{0}$ of 10 , a person positive for the disease will transmit it to an average of 10 people within the community. For any given contagious infectious disease, if $R_{0}<1$, the few infected individuals introduced in a susceptible population have a low probability of infecting others, hence suggesting low disease spread and the possibility of the disease eventually dying out [76]. On the other hand, if $R_{0}>1$, then the infected individuals in a susceptible population will easily infect others thereby increasing the number of infected individuals in each generation [76]. $R_{0}$ can vary significantly depending upon a variety of factors such as the infectious period, probability, and the number of infecting a susceptible individual upon one contact per unit time [75]. The $R_{0}$ value allows one to determine the efforts required to prevent or contain an epidemic from a population. The $R_{0}$ value allows health officials and governments to plan and design strategies to contain a viral outbreak effectively. In summary, a basic reproduction number indicates the risk of viral infection in a community.

The estimated $R$ o value for SARS-CoV-2 has been reported to be around 3.28 with a median of 2.79 and interquartile range of 1.16 according to a study conducted by Liu and colleagues [77]. Another study suggested an estimated $R_{0}$ value of 2.43 to 3.10 across different cities in Italy [78]. On the other hand, an estimated $R_{0}$ value of 2 was accessed during an early outbreak in Wuhan, China [79]. Comparing the $R_{0}$ values across these different studies to the previous SARS-CoV outbreak, the estimated $R_{0}$ values for SARS$\mathrm{CoV}-2$ was found to be slightly higher at $\sim 3$ as compared to the $R_{0}$ values of $\sim 2$ for the previous SARS-CoV outbreak $[77,80]$. Greater $R_{0}$ values indicate that the SARS-CoV-2 has higher transmissibility than the previous SARS-CoV in a susceptible population. The MERS-CoV showed the lowest $R_{0}$ value at 0.7 among the three deadly coronavirus outbreaks, recorded in a study conducted by Petrosillo and colleagues [80]. However, with the ongoing pandemic, it is difficult to obtain an estimate of the SARS-CoV-2 basic reproduction number. Besides, $R_{0}$ values are very subjective to population, data collection, counter preparations, and social customs across the globe as these are the key factors for virus transmission. Hence, the $R_{0}$ values cannot fully represent the outbreak in every country but rather used as a tool to contribute towards an overall understanding of the virus spread.

Given the genetic and structural similarities between the SARS-CoV and SARS-CoV-2, findings on the stability of SARS-CoV-2 are much needed to aid in the control of virus spread. A study has discovered that SARS-CoV-2 exhibits similar surface stability as the SARS-CoV [81]. Another study carried out on multiple $\mathrm{TCID}_{50}$ (median tissue culture infectious dose) revealed the viral titers at which $50 \%$ of the cells were infected. In an aerosolized environment, both SARS-CoV-2 and SARS-CoV showed a similar reduction trend of infectious titre at $10^{3.5}$ to $10^{2.7} \mathrm{TCID}_{50} / \mathrm{L}$ of air and $10^{4.3}$ to $10^{3.5} \mathrm{TCID}_{50} / \mathrm{L}$ of air respectively after $3 \mathrm{~h}[81]$. Also, the SARS-CoV-2 and SARS-CoV exhibited similar stability kinetics where both viruses were more stable on plastic and stainless-steel surfaces than on the copper and cardboard surfaces [81]. On plastic surfaces, both SARSCoV-2 and SARS-CoV showed a significant reduction of virus titre from $10^{3.7}$ to $10^{0.6}$ TCID $_{50}$ and $10^{3.4}$ to $10^{0.7}$ $\mathrm{TCID}_{50}$ after $72 \mathrm{~h}$, respectively. A similar trend was also observed on stainless-steel surfaces after $48 \mathrm{~h}$ where the stability kinetics of SARS-CoV-2 and SARS-CoV reduced from $10^{3.7}$ to $10^{0.6} \mathrm{TCID}_{50}$ and $10^{3.6}$ to $10^{0.6} \mathrm{TCID}_{50}$, respectively [81]. 
A recent study by van Doremalen et al. reported key differences in SARS-CoV-2 and SARS-CoV fomite transmission, which is the transmission of the virus from contaminated surfaces [81]. The viable duration of SARS-CoV-2 was found to be halved on copper surfaces and tripled on the cardboard surfaces compared to the SARS-CoV, at $4 \mathrm{~h}$ and $24 \mathrm{~h}$ viable duration, respectively, a significantly longer survival duration on cardboard surfaces for SARS-CoV-2. The same study also showed that both SARS-CoV-2 and SARS$\mathrm{CoV}$ exhibited similar half-lives in aerosol and on plastic and stainless-steel surfaces [81]. These preliminary results indicate that SARS-CoV-2 has good aerosol and fomite transmission. However, it must be noted that the study was performed with only one SARS-CoV-2 strain. Further studies must be performed to validate the SARS-CoV-2 transmission ability in order to design effective control measures against the virus. Despite SARS-CoV-2 sharing similarities to its predecessor SARS-CoV, it is highly susceptible to mutation and has a much higher transmission capacity. Owing that the basic reproduction number $\left(R_{0}\right.$ value $)$ and transmission stability of SARS-CoV-2 varies across different strains, regions, and continents, it cannot be concluded as a definite value. The effort on basic reproduction number and transmission stability of SARS-CoV-2 are utmost important to illustrate how a different basic reproduction number $\left(R_{0}\right.$ value) and transmission stability can affect the effort to contain the pandemic.

\section{Conclusion}

Several explanations were suggested for the undetermined origin of SARS-CoV-2, in particular the idea of bats, avian and Malayan pangolins as reservoir hosts, owing to the high genetic similarity. As compared to the predecessors, SARS-CoV-2 is much similar to SARS-CoV regarding its general structure and morphology. Key distinctions between the two that contribute to the increased transmissions and severity of COVID-19 reside in the higher binding affinity in SARS-CoV-2 due to the single mutation at N501T in the Spike protein. Spike protein mutations are the most common occurrence among various SARSCoV-2 strains (B.1.1.7, B.1.351 \& P.1), contributing to enhanced binding affinity towards ACE-2 receptors. In addition, SARS-CoV-2 is known for its greater susceptibility to furin cleavage compared to SARS-CoV. Besides, the genetic expression of SARS-CoV-2 differs in the numbers of amino acids in $8 \mathrm{~b}$ and $3 \mathrm{c}$ protein accompanied by the absence of 8 a protein. Moreover, the SARS-CoV-2 encodes for different residues at amino acid position 510 , 723 and 1010, unlike SARS-CoV. Despite the various SARS-CoV-2 strains that are associated with differences in transmission capacity across different continents and regions, the values and data cannot be overlooked as it contributes towards an overall understanding of the virus spread, helping towards the effort to contain the pandemic. Akin to the predecessors, the common symptoms such as fever, dry cough, fatigue, dyspnoea, and headache are also present for SARS-CoV-2. Since SARS-CoV-2 can be largely or mildly asymptomatic upon initial contract, individuals who had close contact with an infected individual or residing in a COVID-19 red-zones should not be excluded for chest CT-imaging and laboratory testing such as RT-PCR. The common findings for COVID-19 are in close resemblance to its counterparts, the SARS-CoV, and MERS-CoV, in which pulmonary lesions, bilateral groundglass opacities, and segmental consolidation are present in lungs on chest CT, a distinctive clinical diagnosis to characterize coronavirus infection.

Author contributions ZYL, AJWY and AS contributed to the preparation and drafting of the manuscript. Critical revisions of the article were done by AS and SKL.

\section{Declarations}

Conflict of interest All authors have read and agreed to the published version of the manuscript. All authors have declared that there is no conflict of interest.

\section{References}

1. WHO (2020) WHO director-general's opening remarks at the media briefing on COVID-19. https://www.who.int/dg/speeches/ detail/who-director-general-s-opening-remarks-at-the-media-brief ing-on-covid-19---3-march-2020. Accessed 20 Dec 2020

2. Worldometer Coronavirus Update (Live). COVID-19 Virus Pandemic. https://www.worldometers.info/coronavirus/. Accessed 6 May 2021

3. Andersen K, Rambaut A, Lipkin W, Holmes E, Garry R (2020) The proximal origin of SARS-CoV-2. Nat Med 26:450-452

4. Zumla A, Hui D, Perlman S (2015) Middle east respiratory syndrome. Lancet 386:995-1007

5. Su S, Wong G, Shi W et al (2016) Epidemiology, genetic recombination, and pathogenesis of coronaviruses. Trends Microbiol 24:490-502

6. Peiris J, Yuen K, Osterhaus A, Stöhr K (2003) The severe acute respiratory syndrome. N Engl J Med 349:2431-2441

7. Alagaili A, Briese T, Mishra N et al (2014) Middle east respiratory syndrome coronavirus infection in dromedary camels in Saudi Arabia. MBio 5:1-6

8. Zaki A, van Boheemen S, Bestebroer T, Osterhaus A, Fouchier $R$ (2012) Isolation of a novel coronavirus from a man with pneumonia in Saudi Arabia. N Engl J Med 367:1814-1820

9. Woo P, Lau S, Huang Y, Yuen K (2009) Coronavirus diversity, phylogeny and interspecies jumping. Exp Biol Med 234:1117-1127

10. Zhu N, Zhang D, Wang W et al (2020) A novel coronavirus from patients with pneumonia in China, 2019. N Engl J Med 382:727-733 
11. Weiss S, Leibowitz J (2011) Coronavirus pathogenesis. Adv Virus Res 1:85-164

12. Saif L (1993) Coronavirus immunogens. Vet Microbiol 37:285-297

13. Xu Z, Shi L, Wang Y et al (2020) Pathological findings of COVID-19 associated with acute respiratory distress syndrome. Lancet Respir Med 8:420-422

14. Thiel V, Herold J, Schelle B, Siddell S (2001) Infectious RNA transcribed in vitro from a cDNA copy of the human coronavirus genome cloned in vaccinia virus The GenBank accession number of the sequence reported in this paper is AF304460. J Gen Virol $82: 1273-1281$

15. Fehr A, Perlman S (2015) Coronaviruses: an overview of their replication and pathogenesis. Coronaviruses 1282:1-23

16. Neuman B, Adair B, Yoshioka C et al (2006) Supramolecular architecture of severe acute respiratory syndrome coronavirus revealed by electron cryomicroscopy. J Virol 80:7918-7928

17. Velavan T, Meyer C (2020) The COVID-19 epidemic. Trop Med Int Health 25:278-280

18. van der Hoek L, Pyrc K, Jebbink M et al (2004) Identification of a new human coronavirus. Nat Med 10:368-373

19. Zhou P, Yang X, Wang X et al (2020) A pneumonia outbreak associated with a new coronavirus of probable bat origin. Nature 579:270-273

20. World Health Organisation. Q\&A on Coronaviruses (COVID-19), WHO international. https://www.who.int/emergencies/diseases/ novel-coronavirus-2019/question-and-answers-hub/q-a-detail/ q-a-coronaviruses\#: :text=symptoms. Accessed 1 Feb 2021

21. Zanin L, Saraceno G, Panciani P et al (2020) SARS-CoV-2 can induce brain and spine demyelinating lesions. Acta Neurochir 162:1491-1494

22. Chan J, Yuan S, Kok K et al (2020) A familial cluster of pneumonia associated with the 2019 novel coronavirus indicating person-to-person transmission: a study of a family cluster. Lancet 395:514-523

23. Wu F, Zhao S, Yu B et al (2020) A new coronavirus associated with human respiratory disease in China. Nature 579:265-269

24. Rothan H, Byrareddy S (2020) The epidemiology and pathogenesis of coronavirus disease (COVID-19) outbreak. J Autoimmune 109:102433

25. Das K, Lee E, Jawder S et al (2015) Acute middle east respiratory syndrome coronavirus: temporal lung changes observed on the chest radiographs of 55 patients. Am J Roentgenol 205:w267-w274

26. Yeo C, Kaushal S, Yeo D (2020) Enteric involvement of coronaviruses: is faecal-oral transmission Of SARS-Cov-2 possible? Lancet Gastroenterol Hepatol 5:335-337

27. Guo M, Tao W, Flavell R, Zhu S (2021) Potential intestinal infection and faecal-oral transmission of SARS-Cov-2. Nat Rev Gastroenterol Hepatol. https://doi.org/10.1038/s41575-021-00416-6

28. Shereen M, Khan S, Kazmi A, Bashir N, Siddique R (2020) COVID-19 infection: origin, transmission, and characteristics of human coronaviruses. J Adv Res 24:91-98

29. Naqvi A, Fatima K, Mohammad $T$ et al (2020) Insights into SARS-Cov-2 genome, structure, evolution, pathogenesis and therapies: structural genomics approach. Biochim Biophys Acta (BBA) - Mol Basis Dis 1866:165878

30. WHO EMROIMERS outbreaksIMERS-CoVlhealth topics. http:// www.emro.who.int/health-topics/mers-cov/mers-outbreaks.html. Accessed 9 Feb 2021

31. Weiss S, Navas-Martin S (2005) Coronavirus pathogenesis and the emerging pathogen severe acute respiratory syndrome coronavirus. Microbiol Mol Biol Rev 69:635-664

32. Letko M, Marzi A, Munster V (2020) Functional assessment of cell entry and receptor usage for SARS-CoV-2 and other lineage B betacoronaviruses. Nat Microbiol 5:562-569
33. Chan J, Lau S, To K, Cheng V, Woo P, Yuen K (2015) middle east respiratory syndrome coronavirus: another zoonotic betacoronavirus causing SARS-like disease. Clin Microbiol Rev 28:465-522

34. Lau S, Woo P, Li K et al (2014) Discovery of anovel coronavirus, china Rattus coronavirus HKU24, from Norway rats supports the murine origin of betacoronavirus 1 and has implications for the ancestor of betacoronavirus lineage A. J Virol 89:3076-3092

35. Ito T, Goto H, Yamamoto E et al (2001) Generation of a highly pathogenic avian influenza A virus from an avirulent field isolate by passaging in chickens. J Virol 75:4439-4443

36. Guo Y, Cao Q, Hong Z et al (2020) The origin, transmission and clinical therapies on coronavirus disease 2019 (COVID-19) outbreak - an update on the status. Mil Med Res 7:1-10

37. Zhou P, Yang X, Wang X et al (2020) Addendum: a pneumonia outbreak associated with a new coronavirus of probable bat origin. Nature 588:E6

38. Wang H, Li X, Li T et al (2020) The genetic sequence, origin, and diagnosis of SARS-CoV-2. Eur J Clin Microbiol Infect Dis 39:1629-1635

39. Kumar S, Maurya V, Prasad A, Bhatt M, Saxena S (2020) Structural, glycosylation and antigenic variation between 2019 novel coronavirus (2019-nCoV) and SARS coronavirus (SARS-CoV). VirusDisease 31:13-21

40. Wan Y, Shang J, Graham R, Baric R, Li F (2020) Receptor recognition by the novel coronavirus from Wuhan: an analysis based on decade-long structural studies of SARS coronavirus. J Virol 94:1-9

41. Zhang $\mathrm{T}, \mathrm{Wu} \mathrm{Q}$, Zhang Z (2020) Probable pangolin origin of SARS-Cov- 2 associated with the COVID-19 outbreak. Curr Biol 30:1346-1351

42. Yamada Y, Liu D (2009) Proteolytic activation of the spike protein at a novel RRRR/S motif is implicated in furin-dependent entry, syncytium formation, and infectivity of coronavirus infectious bronchitis virus in cultured cells. J Virol 83:8744-8758

43. Wang N, Li S, Yang X et al (2018) Serological evidence of bat SARS-related coronavirus infection in humans, China. Virol Sin 33:104-107

44. Belouzard S, Chu V, Whittaker G (2009) Activation of the SARS coronavirus spike protein via sequential proteolytic cleavage at two distinct sites. Proc Natl Acad Sci 106:5871-5876

45. Centers for Disease Control and Prevention (CDC) (2021) Symptoms of Coronavirus. In: Centers for Disease Control and Prevention. https://www.cdc.gov/coronavirus/2019-ncov/symptoms-testi ng/symptoms.html. Accessed 25 Feb 2021

46. Walls A, Park Y, Tortorici M, Wall A, McGuire A, Veesler D (2020) Structure, function, and antigenicity of the SARS-CoV-2 spike glycoprotein. Cell 181:281-292

47. Zheng Y, Ma Y, Zhang J, Xie X (2020) COVID-19 and the cardiovascular system. Nat Rev Cardiol 17:259-260

48. Khatri I, Staal F, van Dongen J (2020) Blocking of the high-affinity interaction-synapse between SARS-CoV-2 spike and human ACE2 proteins likely requires multiple high-affinity antibodies: an immune perspective. Front Immunol 1:1-9

49. Elshabrawy H, Coughlin M, Baker S, Prabhakar B (2012) Human monoclonal antibodies against highly conserved HR1 and HR2 domains of the SARS-CoV spike protein are more broadly neutralizing. PLoS ONE 7:e50366

50. Galloway S, Paul P, MacCannell D et al (2021) Emergence of SARS-Cov-2 B.1.1.7 lineage-United States, December 29, 2020-January 12, 2021. MMWR Morb Mortal Wkly Rep 70:95-99

51. Rose L (2021) B.1.1.7: What We Know About the Novel SARSCoV-2 Variant I ASM.org. https://asm.org/Articles/2021/Janua ry/B-1-1-7-What-We-Know-About-the-Novel-SARS-CoV-2Va\#: :text=All\%20viruses\%20evolve $\% 20$ and $\% 20$ mutate,subst 
itutions\%20or\%20rearrangements\%20of\%20bases. Accessed 21 May 2021

52. Tegally H, Wilkinson E, Giovanetti M et al (2020) Emergence and rapid spread of a new severe acute respiratory syndrome-related coronavirus 2 (SARS-Cov-2) lineage with multiple spike mutations in South Africa. medRxiV. https://doi.org/10.1101/2020.12. 21.20248640

53. Lim H, Baek A, Kim J et al (2020) Hot spot profiles of SARSCov-2 and human ACE2 receptor protein protein interaction obtained by density functional tight binding fragment molecular orbital method. Nat Sci Rep 10:16862

54. Centers for Disease Control and Prevention (CDC) (2021) SARSCoV-2 Variant Classifications and Definitions. https://www.cdc. gov/coronavirus/2019-ncov/cases-updates/variant-surveillance/ variant-info.html. Accessed 21 May 2021

55. Zhang L, Jackson C, Mou H et al (2020) The D614G mutation in the SARS-Cov-2 spike protein reduces S1 shedding and increases infectivity. bioRxiv. https://doi.org/10.1101/2020.06.12.148726

56. Vaduganathan M, Vardeny O, Michel T, McMurray J, Pfeffer M, Solomon S (2020) Renin-angiotensin-aldosterone system inhibitors in patients with covid-19. N Engl J Med 382:1653-1659

57. Li F (2016) Structure, function, and evolution of coronavirus spike proteins. Annu Rev Virol 3:237-261

58. Raj V, Mou H, Smits S et al (2013) Dipeptidyl peptidase 4 is a functional receptor for the emerging human coronavirus-EMC. Nature 495:251-254

59. Li W, Moore M, Vasilieva N et al (2003) Angiotensin-converting enzyme 2 is a functional receptor for the SARS coronavirus. Nature 426:450-454

60. Singh A, Gupta R, Misra A (2020) Comorbidities in COVID19: outcomes in hypertensive cohort and controversies with renin angiotensin system blockers. Diabetes Metab Syndr 14:283-287

61. Hoffmann M, Kleine-Weber H, Schroeder S et al (2020) SARSCoV-2 cell entry depends on ACE2 and TMPRSS2 and is blocked by a clinically proven protease inhibitor. Cell 181:271-280

62. Wang B, Liu Y, Ji C et al (2018) Porcine deltacoronavirus engages the transmissible gastroenteritis virus functional receptor porcine aminopeptidase $\mathrm{n}$ for infectious cellular entry. J Virol 92:1-13

63. Jaimes JA, André NM, Chappie JS, Millet JK, Whittaker GR (2020) Phylogenetic analysis and structural modeling of SARSCoV-2 spike protein reveals an evolutionary distinct and proteolytically sensitive activation loop. J Mol Biol 432:3309-3325

64. Coutard B, Valle C, de Lamballerie X, Canard B, Seidah N, Decroly E (2020) The spike glycoprotein of the new coronavirus 2019-nCoV contains a furin-like cleavage site absent in $\mathrm{CoV}$ of the same clade. Antivir Res 176:104742

65. Angeletti S, Benvenuto D, Bianchi M, Giovanetti M, Pascarella S, Ciccozzi M (2020) COVID-2019: the role of the nsp2 and nsp3 in its pathogenesis. J Med Virol 92:584-588

66. Moura A, Costa H, Correa V et al (2021) Serological assessment of COVID-19 patients in Brazil: levels, avidity, and subclasses of IgG against RBD. Res Sq 1:1-20
67. Qin C, Zhou L, Hu Z et al (2020) Dysregulation of immune response in patients with coronavirus 2019 (COVID-19) in Wuhan, China. Clin Infect Dis 71:762-768

68. Kim J, Heo J, Kim H et al (2017) Neurological complications during treatment of middle east respiratory syndrome. J Clin Neurol 13:227

69. Diao B, Wang C, Tan Y et al (2020) Reduction and functional exhaustion of T cells in patients with coronavirus disease 2019 (COVID-19). Front Immunol. https://doi.org/10.3389/fimmu. 2020.00827

70. Li Z, Yi Y, Luo X et al (2020) Development and clinical application of a rapid IgM-IgG combined antibody test for SARS-CoV-2 infection diagnosis. J Med Virol 92:1518-1524

71. Pan Y, Li X, Yang G et al (2020) Serological immunochromatographic approach in diagnosis with SARS-CoV-2 infected COVID-19 patients. J Infect 81:e28-e32

72. Kaneko N, Kuo H, Boucau J et al (2020) Loss of Bcl-6-expressing $\mathrm{t}$ follicular helper cells and germinal centers in COVID-19. Cell 183:143-157

73. Bauer G, Struck F, Schreiner P et al (2021) The challenge of avidity determination in SARS-CoV-2 serology. J Med Virol 93:3092-3104

74. Bauer G (2020) The variability of the serological response to SARS-corona virus-2: potential resolution of ambiguity through determination of avidity (functional affinity). J Med Virol 93:311-322

75. Dietz K (1993) The estimation of the basic reproduction number for infectious diseases. Stat Methods Med Res 2:23-41

76. van den Driessche P, Watmough J (2008) Further notes on the basic reproduction number. Math Epidemiol 1:159-178

77. Liu Y, Gayle A, Wilder-Smith A, Rocklöv J (2020) The reproductive number of COVID-19 is higher compared to SARS coronavirus. J Travel Med 27:1-4

78. D'Arienzo M, Coniglio A (2020) Assessment of the SARS$\mathrm{CoV}-2$ basic reproduction number, $\mathrm{R} 0$, based on the early phase of COVID-19 outbreak in Italy. Biosaf Health 2:57-59

79. Liu Y, Eggo R, Kucharski A (2020) Secondary attack rate and superspreading events for SARS-CoV-2. Lancet 395:e47

80. Petrosillo N, Viceconte G, Ergonul O, Ippolito G, Petersen E (2020) COVID-19, SARS and MERS: are they closely related? Clin Microbiol Infect 26:729-734

81. van Doremalen N, Bushmaker T, Morris D et al (2020) Aerosol and surface stability of SARS-CoV-2 as compared with SARSCoV-1. N Engl J Med 382:1564-1567

Publisher's Note Springer Nature remains neutral with regard to jurisdictional claims in published maps and institutional affiliations. 